ca, teórica, histórica y antropológica de los cuidados enfermeros. Así como servir de plataforma para el intercambio de estudios, y paradigmas en enfermería.

2. Analizar el aspecto humanista de la enfermería transcultural y su función pedagógica, como nexo de unión entre enfermero y paciente.

3. ${ }^{\circ}$ Promover la reflexión y el debate entre las diferentes concepciones de cuidado a través del tiempo, y sobre todo, poner en marcha una interacción pragmática entre pasado y presente, capaz de situar la enfermería en el espacio social y profesional que merece.

4. ${ }^{\circ}$ Fomentar el pensamiento sociocrítico enfermero potenciando la investigación-acción y el asociacionismo solidario en el marco de la educación para el desarrollo colaborando de forma específica con el Foro de Enfermería para el Desarrollo.

5. Propiciar la actividad literaria en enfermería mediante la organización de un certamen de narrativa titulado "Vida y Salud" que sirva de cauce para desarrollar la antropología narrativa y los aspectos propios de la vertiente fenomenológica en el intervalo salud-enfermedad.

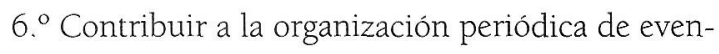
tos (jornadas, congresos) en donde se expongan los trabajos relativos a los campos anteriormente expresados.

7. ${ }^{\circ}$ Promover la edición de una publicación destinada a reflejar todos y cada uno de los anteriores objetivos.

\section{PROGRAMA CIENTÍFICO}

\section{Día 15}

\subsection{0 - Entrega de documentación}

9.30 - Inauguración de las Jornadas a cargo de la Excma. Sra. Ana Laguna. (Vicerrectora de Relaciones Internacionales. Universidad de Alicante)

9.45 - Dra. R. Spector (del Boston College, EE.UU.)

- L"La Enfermería Transcultural: Pasado, Presente y Futuro"

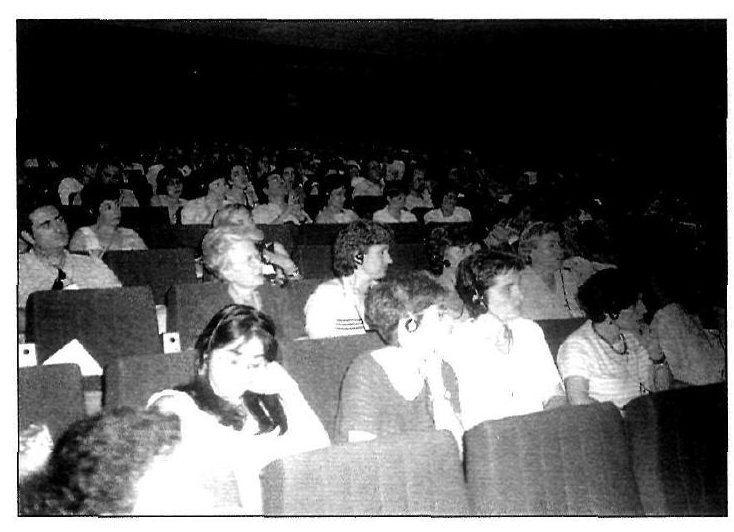

10.25 - Debate

10.50 - Pausa. Café.

11.20 - Presentación de comunicaciones

12.20 - Debate

12.40 - Mesa Redonda: "Antropología de los Cuidados".

Ponentes:

-D. Manuel Amezcua.

-D. Juan Ignacio Valle Racero.

-D. Jorge Mínguez Arias.

13.40 - Debate.

14.00 - Almuerzo de trabajo

17.00 - Dra. M. Gordon (del Boston College, EE.UU)

_-Diagnósticos en Enfermeria: Pasado, presente y futuro"

17.40 - Debate.

18.00 - Pausa. Café.

18.20 - Comunicaciones.

19.20 - Debate

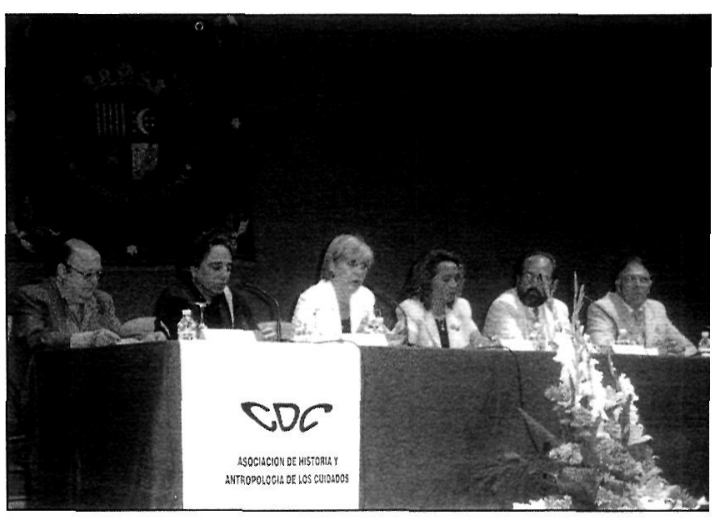



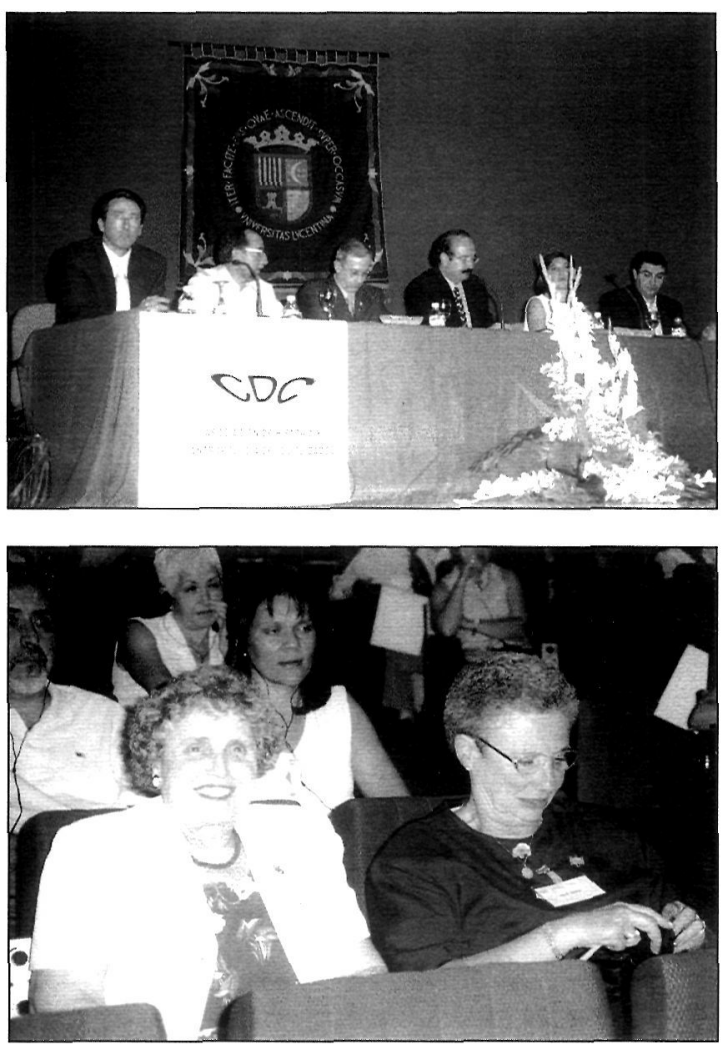

19.40 - Asamblea de la Asociación de Historia y Antropología de los Cuidados.

20.40 - Visita y cóctel en el Castillo de Santa Bárbara. Recital músico-poético: "Amor-Dolor" a cargo de Carolina Terrés y Esther Ferrero.

Día 16

9.30 - Dra. C. Roy (Del Boston College, EE.UU).

- "El Modelo de Adaptación en el contexto de los modelos de enfermería. Dificultades de aplicación."

10.10 - Debate

10.30 - Comunicaciones.

11.30 - Debate

11.40 -Pausa Café.

12.10 - Dr. Fco. Herrera (Universidad de Cádiz)

- Un capítulo de la Enfermería: la cirugía menor en la España del siglo XIX."

12.50. - Dña. C. Chamizo (Enfermera Comunitaria. Gijón)

—"Historia de la Enfermería infantil en Asturias"
13.40 - Debate.

14.00 - Almuerzo de trabajo.

17.00 - Mesa Redonda "Historia, antropología, humanidades y revistas de Enfermería "

Ponentes:

-D. Manuel Amezcua.

-D. Manuel Jesús García Martínez.

-D. Antonio C. García Martínez.

-D. José Siles González.

18.00 - Debate

18.20 - Pausa. Café

18.40 - Comunicaciones

19.40 - Debate

20.00 - Conclusiones y clausura de las Jornadas.

\section{COMUNICACIONES ORALES PARANINFO}

15 de junio de 2000

De 11:20 a 12:20

Modera: Elena Ferrer

- Lo transcultural en enfermería desde un punto de vista terminológico.

Jesús Prieto.

- Acercamiento a la enfermeria transcultural curopea.

Begoña Ruiz de Alegría et al.

- Educación para la Salud en la enfermeria transcultural: un caso práctico, Perú 1996-2000.

Cristina López Osuna et al.

- El análisis de la experiencia como instrumento de control de la práctica profesional y la actividad investigadora en el personal de enfermería. Un estudio etnográfico desde la perspectiva del interaccionismo simbólico.

Encarnación García Hernández.

15 de junio de 2000

De 18:20 a $19: 30$

Modera: Pilar Fernández

-Valoración antropológica como método de adecuación de la actividad en un Centro de Salud. 
Mercedes Rizo Baeza. S. Fernández Ardanaz. -Nuestros Centros de Salud: Lugares Antropológicos.

S. Fernández Ardanaz.

- Una explicación antropológica del embarazo en adolescentes en el campo de Cartagena.

José Antonio Vera Pérez et al.

- El cuidado del adolescente. Un estudio antropológico pendiente en la enfermería.

Manuela Cristina Chas Barbeito.

- Empezando a conocernos. Un acercamiento de la enfermeria al mundo de los jóvenes.

Teresa Ortiz Martínez et al.

16 de junio de 2000

De 10:30 a 11:40

Modera: José Antonio Ávila

-El Modelo de Adaptación: un enfoque práctico.

Manuel Moreno Preciado et al.

-Enseñanza-aprendizaje a los residentes enfermeros:

Los modelos conceptuales a través de la experiencia.

Josefina Ramal López et al.

- El niño y los padres en el Hospital. Cuidados de enfermería.

Miguel Castells Molina.

- Estrategias de dependencia y enfermería.

Ramón Cañamo-Puig et al.

- Guía Clinica de Enfermería: Un buen instrumento de trabajo para planificar los cuidados.

José Sánchez Monfort et al.

16 de junio de 2000

De 18:40 a 19:50

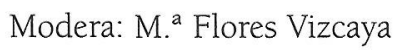

- Alternativas en la enseñanza de la Historia de la Enfermería.

Teresa Salagre de la Vega et al.

-Un pueblo ante la epidemia del siglo XVII

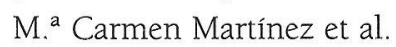

- Hacia donde va la Historia de la Enfermeria.

Carmen González Canalejo.

- ¿Para qué sive la Historia de la Enfermeria?

Mariano Monje Juárez.

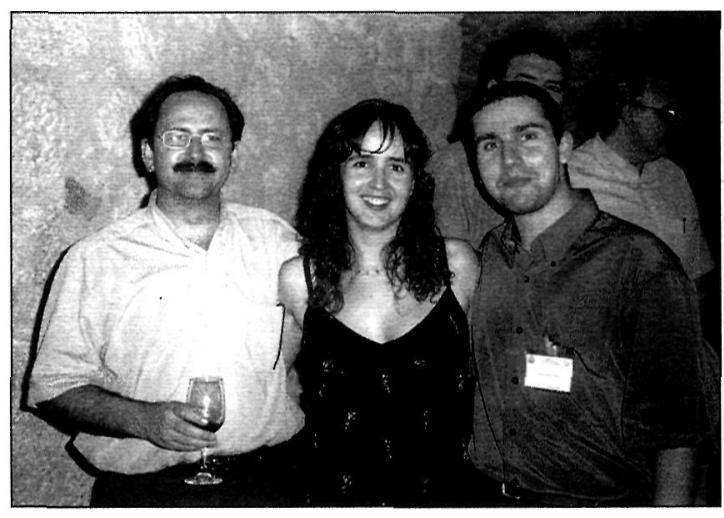

- La Cultura y cuidados en bucodental a principios del siglo XIX.

Francisco Javier Prieto Míguelez.

\section{SALÓN DE GRADOS DE DERECHO}

15 de junio de 2000

De 11:20 a 12:30

Modera: Mercedes Núñez

- Estudio osteométrico de la pelvis de mujer en la población de Sax-Salinas Vs. tipo de parto y bienestar neonatal.

Antonio Penalva Lozano et al.

-Ritos y creencias en el contexto hospitalario.

Rafael Pascual Martínez et al.

- Integración cultural de la educación sobre la muerte en la formación de enfermería.

Joaquín Tomás Sabado y Monserrat Antolín Martín.

- Las prácticas clinicas en Salud Mental como herramienta que posibilita el cambio de actitudes hacia el enfermo mental.

Begoña Ballesteros Benjumea et al.

- "Arrieros somos y en el camino nos encontraremos". Margarita Brugos Flórez et al.

15 de junio de 2000

De 18:20 a 19:30

Modera: Carmen Gloria Molina

- Concebir un hijo en el cambio de milenio: diferencias en el hecho cultural.

Antonia Carricondo y Manuel Amezcua. 
- "Un rito en el modelo de cuidados de los Vaquiros de Alzada".

Manuel Angel Martínez et al. - Viviendo la teoría enfermera: estudio fenomenológico sobra la experiencia vivida en el proceso de adopción y uso de un modelo de cuidados.

Carmen Arribas Barahona et al. - Las enfermeras frente a las necesidades religiosas de los pacientes: estudio fenomenológico de la experiencia de las enfermeras acerca de las necesidades espirituales de enfermos hospitalizados.

Inmaculada Ceballos Benito.

- Reflexiones sobre el cuidado de una adolescente con problemas de salud.

Purificación Cabezas Yáñez y Anna Ramiró Jofre.

16 de junio de 2000

De $10: 30$ a $11: 40$
Modera: Asunción Ors

- Estudio comparativo sobre el juicio profesional enfermero (Hailsham II Ward del District General Hospital. UK vs. Hospital de Txagorritxu. Vitoria).

Estíbaliz Cristóbal Domínguez.

- Estudio comparativo sobre la participación del paciente en los cuidados de Enfermeria.

Ainara López Palenzuela.

-Opinión de las mujeres sobre la menopausia, un estudio cualitativo.

Ángel Villalva et al - El cuidador principal en el núcleo familiar catalizador.

Arturo Morell Alemany et al.

-Cuidados Paliativos al final de la vida.

M. a Ángeles García-Carpintero et al.

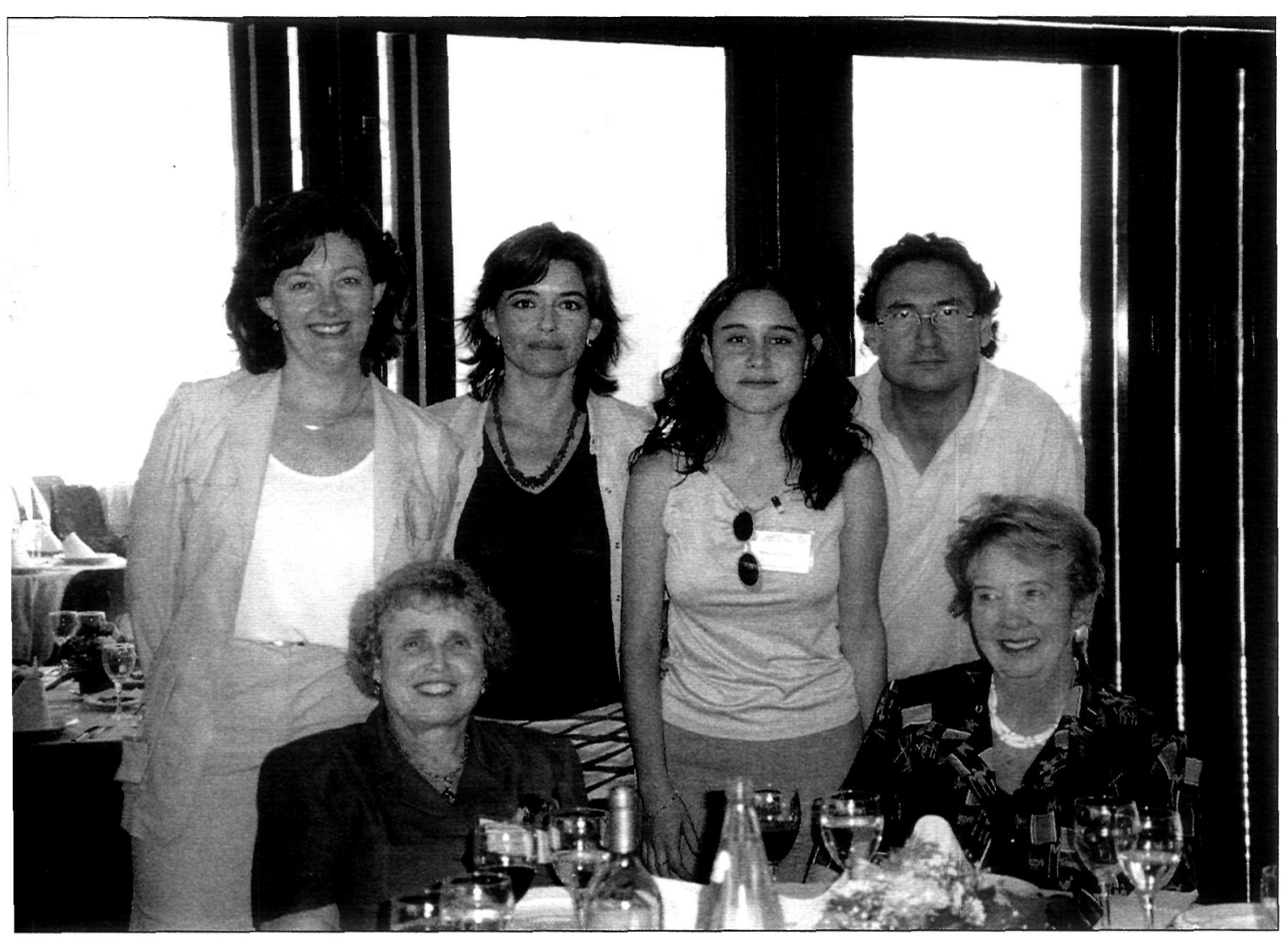


16 de junio de 2000

De 18:40 a 19:10

Modera: Eva M. ${ }^{a}$ Gabaldón

- Diseñadoras de estilos profesionales: Una pasarela histórica.

\section{A. Martín Marino et al.}

- Cultura del trabajo: enfermeras en unidades especiales.

Mercedes Núñez et al.

-Desarrollo del conocimiento en enfermería.

Ramón Camaño-Puig.

- Apoyo al diagnóstico de autoconcepto en Atención Primaria.

Ana García Laborda.

\section{POSTERS (defensa oral)}

DÍA 15

11:30 Proyecto de formación para alumnos de FP rama sanitaria sobre prevención sobre enfermedades cardiovasculares. M. ${ }^{2}$ Dolores Nonay et al.

11:40 Cuidando a la comunidad: Intervención comunitaria en prevención de enfermedades cardiovasculares. M. ${ }^{a}$ Dolores Nonay et al.

11:50 La religión, su influencia en los cuidados enfermeros. M.V. Orejón

12:00 Actividad que realiza el equipo de enfermeria para preservar la intimidad de la persona ingresada. Susana Cariñanos.

12:20 Evolución histórica de las matronas en España.

A. Manchado et al.

18:10 Desarrollo de los Talleres de Cuidado de Enfermeria en Pacientes con Fibromialgia en la Consulta de Reuma del Hospital de Alcoy. M. ${ }^{a}$ Dolores Gil del Gallego.

18:20 Construyendo una enfermería europea a través del programa ENI. Elena de Lorenzo et al.

18:30 Trabajar con un modelo de Enfermeria: esbozo de un proyecto. Purificación González et al.

18:40 Aplicación de planes de cuidados en el despertar. Registros. A. Calvo et al.
18:50 Creencias mágico-religiosas relativas a la placenta y al cordón umbilical. M. ${ }^{a}$ Dolores Estellés Morant.

19:00 La escucha: una herramienta de Enfermería. Jesús Prieto et al.

19:10 La familia como proveedora de cuidados. Joaquín Lima et al.

19:20 Experiencia transcultural: programa ENI. Jagoba Zarandona et al.

\section{DÍA 16}

10:30 "...Queda la música...". M. ${ }^{a}$ Consuelo Carballal et al.

10:40 Historia de la enfermería psiquiátrica en Gran Canaria. Josefina Ramal López et al.

10:50 Sistemas de transporte al servicio de los cuidados enfermeros. Aurora Baraza et al.

11:00 Valores sociales y cuidados. Antonia M. ${ }^{2}$ Tomás et al.

11:10 Atención a los pacientes oncológicos terminales desde una UHD. M. ${ }^{2}$ A. García-Carpintero el al.

11:20 El arte de morir, luz de un sentimiento. BlancaFlor Eiranova y Paloma-Rosa Eiranova.

11:30 El mal del bacilo de Koch. Blanca-Flor Eiranova y Paloma-Rosa Eiranova.

18:40 Las dimensiones de la enfermedad. A propósito de dos etnografias sobre los nervios. Eva Fernández y Montse Masip.

18:50 El grupo familiar valorado por enfermería a través de los patrones funcionales. M. ${ }^{a}$ Consuelo Carballal et al.

19:00 Enfermeria basada en la evidencia (I): Creación de un instrumento útil. Rita Pulido et al.

19:10 Enfermería basada en la evidencia (II): Cómo utiliza la enfermera el dossier de cuidados. Rita Pulido et al.

19:20 Enfermeria basada en la evidencia: Experiencia piloto del proyecto Enfermera responsable-Enfermera colaboradora. Rita Pulido et al. 


\section{COMITÉS}

COMITÉ ORGANIZADOR:

Presidenta de las Jornadas

Ilustrísima Señora. Loreto Maciá.

Directora de la E.U. E. de Alicante.

Coordinadores Generales

Sr. Dn. José Siles González

Sr. Dn. Luis Cibanal Juan

Sr. Dn. Juan Mario Domínguez

Vocales

Gabaldón, Eva

Calle, José M

Gracia, María

Migallón, Belén

Migallón, María

López, Francisco

Domingo, Manuela

Beltrá, Miriam

Gómez, Javier

López, Marisa

López, Cristina

Rodríguez, Olivia

Vizcaya, M. Flores
Coordinador General

Sr. Dn. José Siles González

Vocales

Maciá, Loreto

Ávila, José A.

Cibanal, Luis

Domínguez, Mario

Esteban, Belén

Fernández, Pilar

Figuérez, Juan

Galao, Roberto

Lillo, Manuel

Solano, M. Carmen

Monge, Mariano

Núñez, Mercedes

Pérez, Rosa

Rizo, Mercedes

Sanz, M. ${ }^{a}$ Isabel

Siles, José

Verdú, Antonio

Vizcaya, M. Flores

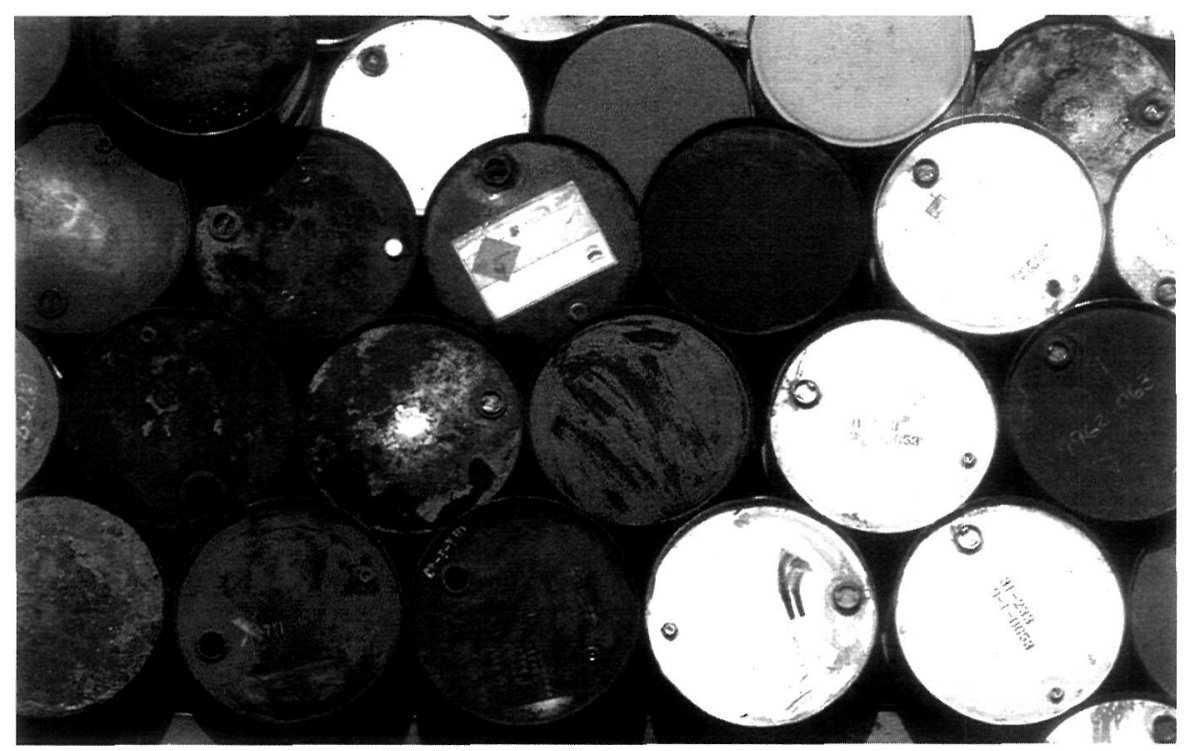

